\title{
Introduction to Selected Contributions from the 53rd US Rock Mechanics/Geomechanics Symposium Held in New York, June 23-26, 2019
}

\author{
Mahdi Heidari ${ }^{1}$. Herbert H. Einstein ${ }^{2}$
}

Published online: 5 July 2021

(c) The Author(s), under exclusive licence to Springer-Verlag GmbH Austria, part of Springer Nature 2021

This special issue contains papers selected from contributions submitted to the 53rd US Rock Mechanics/Geomechanics Symposium held in New York on June 23-26, 2019. This symposium, held annually by the American Rock Mechanics Association (ARMA), is one of the few major events worldwide to share the-state-of-the-art research and practice in all aspects of rock mechanics and rock engineering. Presented works include qualified recent theoretical and applied studies conducted by scientists in research labs, professional engineers from industry and government, and students from academia.

The first paper in this issue is the part of the MTS keynote lecture about fractures presented by Professor Einstein at the 2019 ARMA symposium. He repeats the observation cycle from the large (field) scale to increasingly detailed laboratory scales of fractures and shows that fractures determined as tensile or shear on the large scale are indeed produced through a combination of both tensile and shear mechanisms on the smaller scale. A variety of techniques involved in the observations are briefly described. This work highlights the importance of simultaneous observation and modeling and avoiding overgeneralization of the findings.

The rest of the papers in this issue have been selected from hundreds of papers presented at the symposium through a rigorous evaluation process. They were nominated first by reviewers, then session chairs and track leads, and finally by the symposium award committee. Evaluation of the studies was based on the quality of both the technical

Mahdi Heidari

mahdiheidari@utexas.edu

Herbert H. Einstein

einstein@mit.edu

1 Bureau of Economic Geology, Jackson School of Geosciences, University of Texas at Austin, Austin, USA

2 Department of Civil and Environmental Engineering, Massachusetts Institute of Technology, Cambridge, USA content and the presentation and discussion of the study. The selected papers were peer-reviewed again by subject-matter experts to be expanded and improved to meet the standards of the special issue.

Papers in this issue have been selected in four fields of Civil, Mining, Petroleum, and Interdisciplinary Engineering. There are two papers in the field of Civil engineering. Gutiérrez et al. (2021) conduct 3D DEM models of axially loaded rock-socketed piles to investigate the role of the socket roughness on the side shear resistance of the piles. The models, calibrated with experimental data, successfully predict settlement, side shear resistance, and the damage at the interface of a rock-socketed pile. Based on the results of the numerical models, an empirical factor is introduced for the estimation of the side shear resistance of the piles based on the pile roughness and the uniaxial compressive strength (UCS) of the rock. The results of this study indicate that the pile roughness significantly affects the pile behavior and should thus be incorporated in the design practice of these piles.

Laouafa et al. (2021) develop a constitutive model to describe the mechanical behavior of dissolving rocks such as gypsum, limestone, and halite. The model is developed based on the microscale multiphysics and then formulated in macroscale. The model is employed to study the subsidence induced by the dissolution of one or more gypsum lenses and the stability of the covering and the failure of a gypsum pillar in an abandoned quarry.

This issue has two papers in the field of Mining engineering. Tuncay et al. (2021) use recent stress measurements in retreat room-and-pillar mines with different depths and mining dimensions to determine the stable abutment angle. They find that the value currently assumed in the design practice $\left(21^{\circ}\right)$ holds true only for mines shallower than $200 \mathrm{~m}$; in deeper mines, the stable angle is lower, decreasing with depth. Finite-volume models show that the abutment angle is lower in deep mines because shallow panel gobs support 
overburden and thereby decrease the load on deeper panels. A new equation is proposed for the stable abutment angle that is valid in both moderate and deep mines.

Williams et al. (2021) use new techniques to reprocess InSAR monitoring data that were collected at the Bingham Canyon Mine, Lake City, Utah, before a large landslide occurred at the upper northeast wall of the pit in 2013. Their analysis reveals that significant ground movements had occurred for several years prior to the failure. This finding would have led to different slope management and mine development plans and have possibly prevented the failure. Their study highlights the significance of new InSAR processing methods.

Four papers are from the field of Petroleum Engineering. Song et al. (2021) develop a phenomenological 1D model for percussive drilling systems to investigate why maximum rate of penetration occurs at an optimal weight-on-bit. The 1D model assumes that the impact of the hammer generates a longitudinal wave in the bit. This study demonstrates that the energy transmitted from the bit to the rock depends on the pseudo-stiffness of the rock-bit interface. The dependence of this stiffness on the weight-on-bit explains the existence of an optimal weight-on-bit for maximum rate of penetration.

Zhang et al. (2021) use the in situ Small-Angle Neutron Scattering (SANS) technique to study the evolution of nanopores in a coal specimen under increasing uniaxial compression. They use porosity invariant mapping to quantify apparent porosities at different azimuthal angles and structure-free orientation factors such as the alignment factor and Hermans' orientation factor to quantify the orientation of nanopores. They find that apparent porosity is higher along the coal bedding direction, the orientation of pores toward the coal bedding direction increases with the pore size, and up to a stress of 575 bar, no distinct porosity modification is observed after a stress relief.

Perbawa et al. (2021) assess the error in data measured in triaxial tests due to the roughness of the specimen endcap surfaces. Different materials including aluminum, shale, sandstone, and carbonate are tested at different test conditions. The endcap-surface roughness introduces error in cap-to-cap-based measurements of stiffness, Poisson's, and Biot's ratios, magnifies biases caused by the specimen bending and tilting, and hinders ultrasonic energy transmission. The error is reduced by using higher confining pressure or specimen-bonded strain gauges.

Bui (2021) develops a new multi-phase flow model to evaluate the effects of salinity, fluid type, shale swelling, and wettability alteration on oil recovery from water injection in unconventional reservoirs. A mass-transport model is coupled with a geomechanical model to account for both the mass exchange between the matrix and the fractures and the fluid interaction with the rock permeability and porosity. This study shows that osmosis significantly contributes to the longterm oil recovery from low-permeability shale matrix, and that shale-matrix swelling significantly reduces the permeability of the matrix and fractures and thereby reduces overall oil recovery. The results suggest that water injection is inappropriate for shale with high swelling potential.

There are three papers in the Interdisciplinary field. Maedo et al. (2021) extend the Mesh Fragmentation Technique (MFT) for non-isothermal evolution of fractures in rocks. MFT is a finite-element technique that enables the finite-element codes to model fractures by incorporating High-Aspect-Ratio (HAR) elements between standard finite elements. The extended method successfully reproduces available analytical, experimental, and numerical results. This work expands the application of the method to non-isothermal problems such as high-level nuclear waste disposal, hydraulic fracturing in conventional oil reservoirs, and Enhanced Geothermal Systems.

Zhou et al. (2021) develop a constitutive model for fractured sorptive geomaterials such as coals to estimate the impact of gas production from these rocks on their deformation and permeability. The model considers the fracture/cleat spacing, in-situ stress condition, and the matrix sorptive property. The model is encoded and used in a finite-element model to simulate methane depletion in a coal specimen. The model is validated against methane and helium pressurization tests on San Juan basin coals. It is shown that the coal permeability can decrease or increase depending on the competition between desorptioninduced fracture opening and poroelastic compaction.

Gheibi et al. (2021) monitored the slippage of a natural sandstone joint in a simple-shear test with both digital imaging and ultrasonic wave measurements to identify ultrasonic precursors associated with the slippage of these joints. Fractured sandstone joints from Yunnan China were sheared up to reach the steady-state post-peak (residual) stress and then the localized slippage along the joint surface were monitored simultaneously by multiple ultrasonic $\mathrm{P}$ and $\mathrm{S}$ wave transducers and digital image sensors. They documented two failure modes of slow and dynamic and for each mode three phases of inter-seismic, pre-seismic, and co-seismic. Their results show that seismic precursors obtained based on the amplitude of converted ultrasonic waves are closer to the peak shear stress. This study provides a new insight into the evaluation of slippage mechanisms of rock joints based on ultrasonic waves.

In the end, I would like to emphasize that this special issue would not have been produced without the voluntary dedication of reviewers and editors of the symposium and of the Rock Mechanics and Rock Engineering journal. I particularly thank Mariacristina Bonini, Narmadha Purusothaman, Herbert Einstein, Giovanni Barla, and Jean Sulem for their continued assistance and advice during the production of this issue.

Publisher's Note Springer Nature remains neutral with regard to jurisdictional claims in published maps and institutional affiliations. 